\title{
Star formation on galactic scales
}

\author{
Robert C. Kennicutt, Jr. ${ }^{1,2}$ \\ ${ }^{1}$ Institute of Astronomy, University of Cambridge, Madingley Road, Cambridge CB3 0HA, UK \\ email: robk@ast.cam.ac.uk \\ ${ }^{2}$ Steward Observatory, University of Arizona, Tucson, AZ 85721, USA
}

\begin{abstract}
New multi-wavelength data on nearby galaxies are providing a much more accurate and complete observational picture of star formation on galactic scales. Here I briefly report on recent results from the Spitzer Infrared Nearby Galaxies Survey (SINGS). These provide new constraints on the frequency and lifetime of deeply obscured star-forming regions in galaxies, the measurement of dust-corrected star formation rates in galaxies, and the form of the spatiallyresolved Schmidt law.
\end{abstract}

Keywords. galaxies: ISM, infrared: galaxies, stars: formation

\section{Introduction}

One of the least understood facets of the broader problem of star formation has been the triggering and regulation of star formation on galactic scales. The well-defined patterns in star formation within galaxies and along the Hubble sequence provide clear qualitative evidence for the importance of large scale drivers (e.g., Kennicutt 1998a). However our quantitative understanding of these processes has been hampered by the complexity of the ISM on these scales, and until recently by the relative dearth of accurate observations of star formation and the ISM in other galaxies. Now thanks to a flood of new observations from the Galaxy Evolution Explorer (GALEX), the Spitzer Space Telescope, and groundbased telescopes we are constructing accurate maps of star formation in galaxies for the first time. These are enabling statistical studies of star formation rates (SFRs) for well-defined samples of hundreds to thousands of galaxies, along with in-depth spatially-resolved studies of the nearest galaxies, often incorporating high-resolution maps of the atomic and molecular gas.

Space does not allow for a complete discussion of even the most recent work, so this paper will highlight only a few results with emphasis on one project, the Spitzer Infrared Nearby Galaxies Survey (SINGS). SINGS is one of the six original Spitzer Legacy surveys. We have used the Spitzer telescope to image 75 nearby $(d \leqslant 30 \mathrm{Mpc})$ galaxies at $3.5-160 \mu \mathrm{m}$, and obtain spectra maps of the galaxies including their centers and 80 extranuclear star-forming regions. These are supported by ancillary observations ranging from the ultraviolet (deep GALEX imaging) through the visible and near-infrared, to the radio continuum, HI, and CO. Although SINGS was primarily designed to provide a general-purpose multi-wavelength archival resource for studying the structure and evolution of these galaxies, its core science program has been aimed at studying the star formation and ISM properties of the Hubble sequence. A description of the survey and its science goals can be found in Kennicutt et al. (2003). Here I highlight a few results that are especially pertinent to the other results presented at this conference, including the statistics of highly-obscured star forming regions and the star formation (Schmidt) law in galaxies. 


\section{Infrared probes of star formation in galaxies}

Dust extinction has long been one of the limiting factors in measurements of SFRs of galaxies, locally and at high redshift. The shape of the cosmic background shows us that approximately half of the ultraviolet and visible starlight in the universe is absorbed and re-emitted in the infrared, so any census of star formation in either the UV/visible or the infrared is bound to be missing half of the star formation on average, with enormous variations from object to object.

The Spitzer telescope has enabled an important breakthrough in this problem, by enabling us to map galaxies in the thermal infrared with an angular resolution that is of the same order as imaging in the ultraviolet and visible. For nearby galaxies $(d<30 \mathrm{Mpc})$ this corresponds roughly to the linear scales over which dust reprocesses the starlight and the scale on which molecular cloud formation is triggered. As a result we have devoted considerable effort in SINGS to using the combination of infrared, ultraviolet, and $\mathrm{H} \alpha$ measurements to derive robust extinction-corrected maps of the star formation.

Figure 1 illustrates the primary diagnostics that are available to us, the photospheric emission of stars in the near-ultraviolet $(150$ and $250 \mathrm{~nm}$ ) from the GALEX telescope, the nebular-processed far-ultraviolet emission in $\mathrm{H} \alpha$, and two tracers of the dust emission at $8 \mu \mathrm{m}$ and $24 \mu \mathrm{m}$. The $8 \mu \mathrm{m}$ emission is dominated by molecular "PAH" band features, and includes discrete components from the neutral envelopes around HII regions and other star-forming regions, along with a more diffuse component (evident as the arms and filaments in M81) that represents dust in the general interstellar medium being excited by the interstellar radiation field. In contrast the $24 \mu \mathrm{m}$ emission is dominated by silicate grains, and the positions of the infrared peaks are strongly correlated with optical HII regions, both in position and in flux. There is also a more diffuse, cooler component that dominates at longer wavelengths, the "infrared cirrus" that can now be resolved from the discrete sources directly with Spitzer.

Calzetti et al. (2005) and Pérez-González et al. (2006) have studied the star formation regions in M51 and M81 in depth, and were able to show that the $24 \mu \mathrm{m}$ luminosities of the HII regions are strongly correlated with the dust-obscured ionizing fluxes. This encouraged us to calibrate a composite SFR measure based on a weighted sum of the $\mathrm{H} \alpha$ and $24 \mu \mathrm{m}$ luminosities to provide an extinction-corrected estimate of the $\mathrm{H} \alpha$ and ionizing fluxes. The same approach has already been applied to the combination of UV and total infared fluxes of star-forming regions in the "flux ratio method" of Gordon et al. (2000), and we simply have extended the approach to $\mathrm{H} \alpha$. In the same way an approximate $\mathrm{H} \alpha$ extinction can be estimated from the ratio of $24 \mu \mathrm{m}$ to $\mathrm{H} \alpha$ fluxes. The promise of this technique is illustrated in Figure 2. In the left panel we have compared extinctioncorrected $\mathrm{H} \alpha$ luminosities for $240 \mathrm{HII}$ regions in a sample of SINGS galaxies using this method to independently corrected luminosities based on $\mathrm{P} \alpha / \mathrm{H} \alpha$ ratios. The method is robust for statistical samples of objects, but there is a dispersion of approximately \pm 0.2 dex for individual regions, which probably reflects variations in extinction geometry and dust heating along with age and upper mass function variations in the clusters. The right panel shows a similar comparison, but in this case applied to entire galaxies. There we have combined IRAS and $\mathrm{H} \alpha$ fluxes to derive an extinction-corrected SFR, and compared it to SFRs corrected using integrated spectroscopic measurements of the $\mathrm{H} \alpha$ and $\mathrm{H} \beta$ lines, from Moustakas \& Kennicutt (2006). Again the correlations are not perfect, but the method appears to offer a reasonably accurate means of correcting for extinction for galaxies with attenuations ranging from zero to a few magnitudes. 

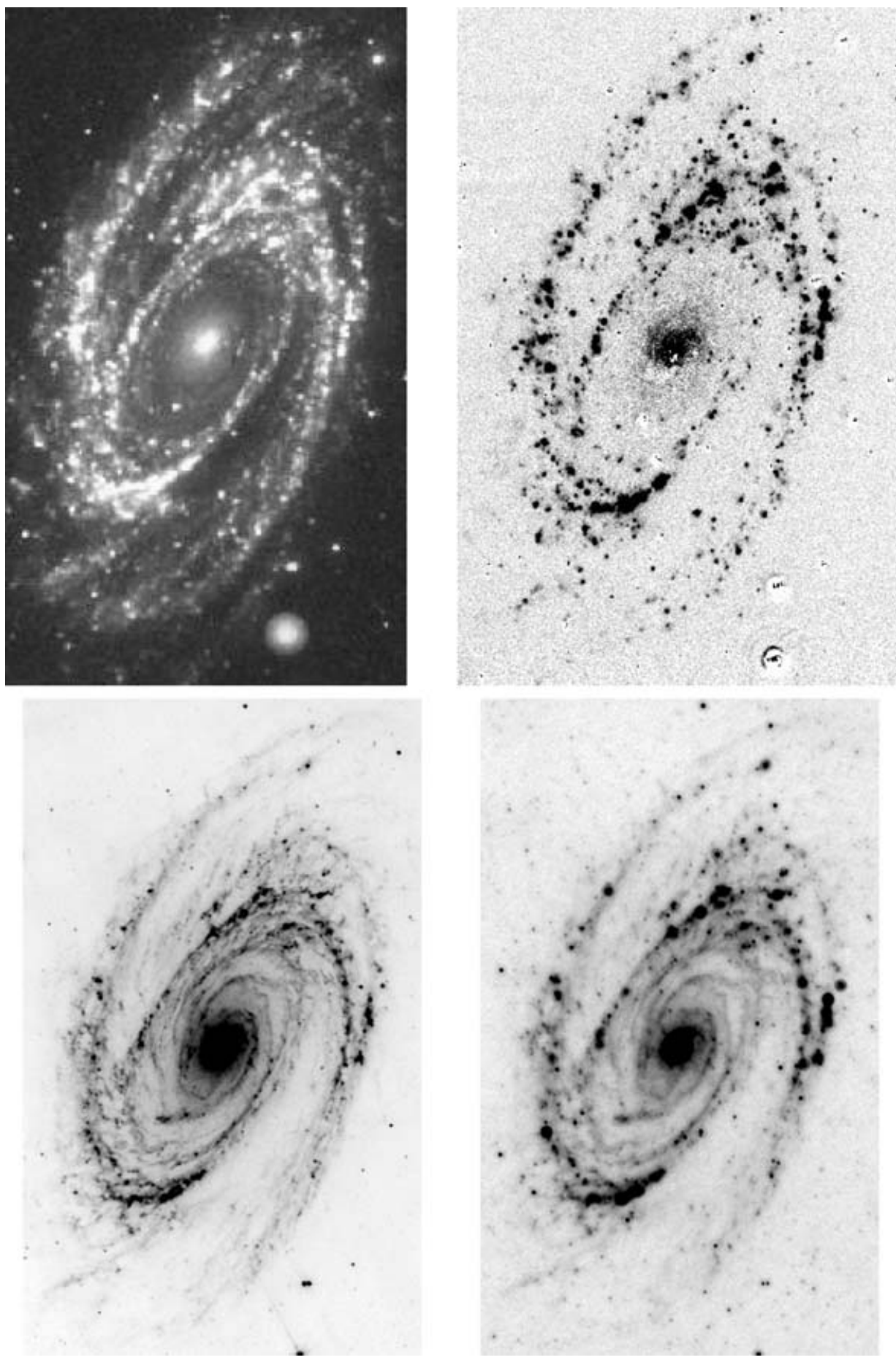

Figure 1. The nearby Sab spiral M81 (NGC 3031) observed at four wavelengths that provide complementary tracers of massive star formation and the associated ISM. Top left: Utraviolet $(1350-2800$ A), imaged with the GALEX satellite. Top right: M81 in H $\alpha$, observed with the Burrell Schmidt telescope, courtesy R. Walterbos. Lower Left: $8 \mu \mathrm{m}$ with the IRAC camera on Spitzer. Lower right: $24 \mu \mathrm{m}$, as imaged with the MIPS instrument on Spitzer. Note the dramatic change in the structure and the clumpiness of the infrared emission between $8 \mu \mathrm{m}$ (PAH dominated) and $24 \mu \mathrm{m}$ (silicate dust dominated), and the presence of diffuse "cirrus" emission at both infrared wavelengths. 

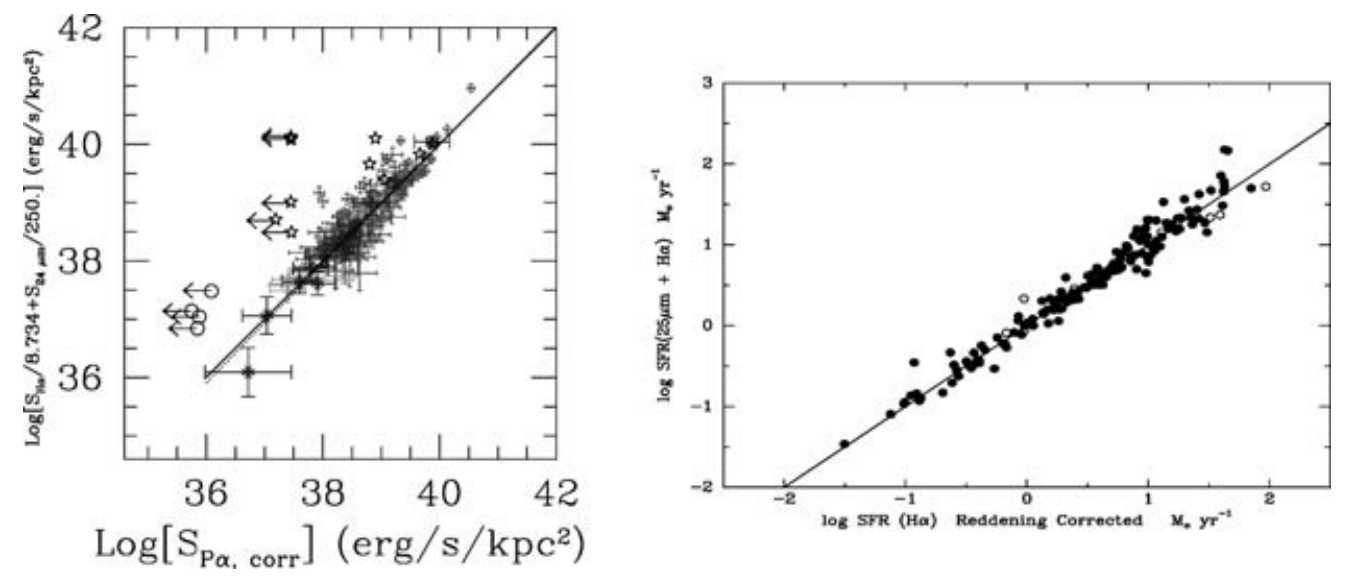

Figure 2. Left: Comparison of extinction-corrected $\mathrm{H} \alpha$ luminosities of HII regions based on a combination of $24 \mu \mathrm{m}$ and $\mathrm{H} \alpha$ fluxes with independently corrected fluxes based on $\mathrm{P} \alpha$ and $\mathrm{H} \alpha$ data. Right: A similar comparison for entire galaxies, compared this time to independently corrected SFRs from integrated optical reddenings.

\section{Dust-obscured star-forming regions}

One of the surprising early results in the SINGS imaging was the rarity of bright infrared sources without $\mathrm{H} \alpha$ and/or ultraviolet counterparts. This can be seen clearly in Figure 1 by comparing the $24 \mu \mathrm{m}$ and $\mathrm{H} \alpha$ panels in particular; there is nearly a one-to-one correspondence between regions. This contrasts sharply with the results from infrared surveys of star-forming regions on smaller spatial and luminosity scales, where deeply embedded and optically invisible sources represent a significant fraction of the population.

These results have been quantified in a study by Prescott et al. (2006, in preparation). Those authors have obtained infrared and $\mathrm{H} \alpha$ photometry for the well-resolved source populations in more than 50 of the SINGS galaxies, and investigated the statistics of the optical to infrared colors of these objects. Using the methods described earlier to estimate extinctions from the ratio of $24 \mu \mathrm{m}$ to $\mathrm{H} \alpha$ fluxes, Prescott et al. find that more than $96 \%$ of the infrared sources have $\mathrm{H} \alpha$ counterparts with extinctions (averaged across a beam diameter of $500 \mathrm{pc}$ ) of $A_{V}<4$ mag. This contrasts sharply with the deeply embedded and even more luminous regions that are often found in the circumnuclear regions of infrared-luminous starburst galaxies. Indeed in our sample the small fraction of highly obscured objects tends to be concentrated in the central regions of the SINGS galaxies, though examples at large radii are not infrequent.

Of course this in no way implies that the familiar population of compact and ultracompact HII regions with high extinctions are absent in the SINGS galaxies! The average extinction of the star-forming regions is roughly 1 magnitude, so one could contrive a model in which $60 \%$ of the young stars in our 500 pc beams were completely invisible, and the other $40 \%$ were dust-free, and it would fit our typical observation. I suspect that after more complete study of individual regions in the most well-resolved galaxies that the contribution of deeply embedded stars will turn out to be much lower, but certainly it does not have to be as low as $4 \%$. However our results do demonstrate that very luminous embedded clusters are rare - such objects must be able to clear channels for optical radiation to escape on relatively rapid timescales (order $10^{5}-10^{6} \mathrm{yr}$ ) compared to the typical ionizing lifetimes of such cluster. The relative rarity of infrared sources 

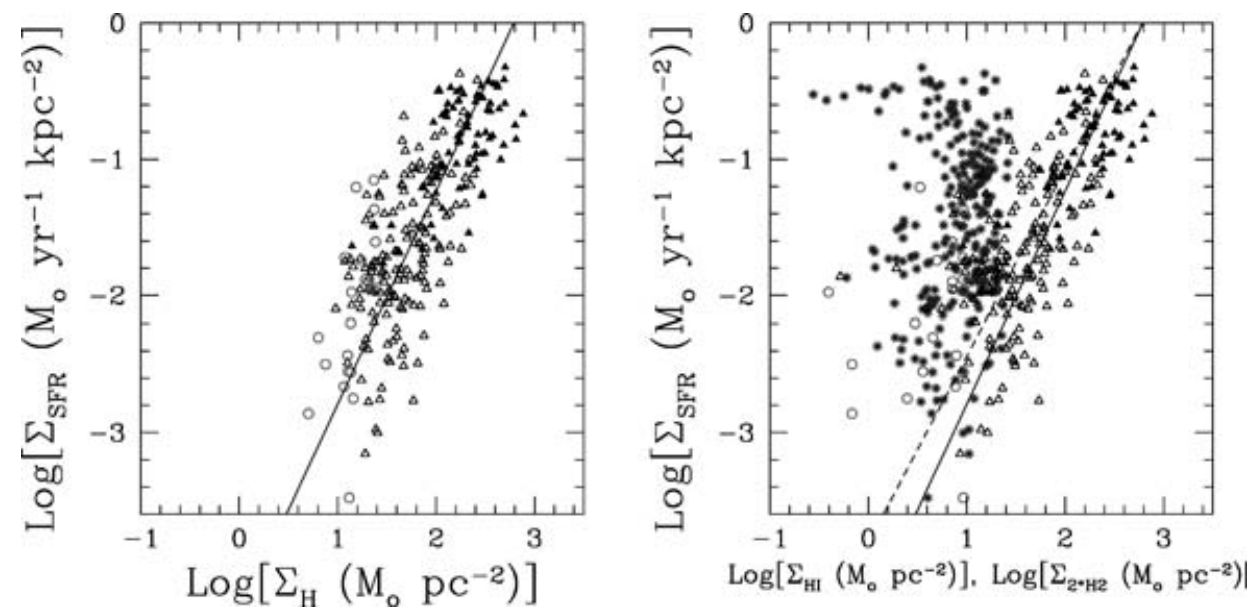

Figure 3. Schmidt law for 257 star-forming regions in M51. Left: The SFR surface density is plotted as a function of total gas surface density. Right: The SFR density is plotted as functions of the $\mathrm{H}_{2}$ surface density alone (points) and $\mathrm{HI}$ surface density alone (stars).

without HII regions also places interesting constraints on the lifetimes of the natal dust clouds. If the parent clouds remained close to the OB stars for much longer than the lifetimes of HII regions (order $\sim 5 \mathrm{Myr}$ ), we would expect to see many bright dust regions with bright near-ultraviolet counterparts but little or no $\mathrm{H} \alpha$ emission. The rarity of such objects suggests that the dust dissipates on roughly the same timescale as the ionizing lifetime, consistent with results from kinematic measurements of the HI shells about OB associations presented earlier in this symposium.

\section{The spatially-resolved star formation law}

I conclude with an illustration of what can be learned by applying these multi-wavelength techniques. The ability to measure extinction-corrected SFRs across a galaxy makes it possible to study the behavior of the SFR density vs gas density law (Schmidt law) on a point by point basis in galaxies. It is now known that on global scales the disk-averaged SFR and gas densities of disks are well fitted by a Schmidt power law relation with slope $N \simeq 1.4$ (Kennicutt $1998 \mathrm{~b}$ ). However the global relation offers few clues to the physical underpinnings of the star formation law, and other prescriptions, for example a parametrization of the SFR density in terms of the ratio of gas density to local dynamical time fits the disk-averaged properties of galaxies nearly as well (Kennicutt 1998b).

As a pilot study of this application we have combined our SINGS infrared and $\mathrm{H} \alpha$ observations of M51 with CO data from the BIMA SONGS survey (Helfer et al. 2003) and the THINGS HI survey (Brinks et al., this volume), to correlate the SFR density with the local atomic, molecular, and total cold gas surface densities, on scales ranging from $520 \mathrm{pc}$ (the effective resolution limit of our data) to about $2 \mathrm{kpc}$. Some of the key results are shown in Figure 3, which shows the SFR vs gas density relations for 257 star-forming regions. The left panel shows the correlation with total gas density, and reveals a Schmidt law with slope $N=1.55 \pm 0.1$, where our uncertainty includes a conservative allowance for systematic effects. This is similar to the global law observed in disks $(N \sim 1.4)$, and certainly rules out a linear dependence, as one might expect if the ratio of stars to gas (SFE) in the regions was constant independent of mass scale. In the right panel we plot the SFR densities against the molecular and atomic gas surface densities separately. The 
disk of M51 is overwhelmingly molecular, especially in the star-forming clouds, so the correlation of SFR density with $\mathrm{H}_{2}$ surface density is nearly the same as in the left panel (confirming work on M33 by Heyer et al. 2004). Interestingly there is no evidence of a correlation with HI surface density at all; the most prominent feature in the panel is a rather sharp upper cutoff to the observed HI column densities, which may signal the optical depth limit for the efficient conversion to molecular gas. We intend to extend this work to the other galaxies in the SINGS sample with high-quality $\mathrm{CO}$ and HI data.

\section{Acknowledgements}

Support for the SINGS project, part of the Spitzer Space Telescope Legacy Science Program, is provided by NASA through Contract Number 1224769 issued by JPL, Caltech, under NASA contract 1407. This work was also supported in part by NSF grant AST-0307386.

\section{References}

Calzetti, D., et al. 2005, ApJ 633, 871

Dale, D.A., et al. 2005, ApJ 633, 857

Gordon, K.D., Clayton, G.C., Witt, A.N. \& Misselt, K.A. 2000, ApJ 602, 723

Helfer, T.T., et al. 2003, ApJS 145, 259

Heyer, M.H., Corbelli, E., Schneider, S.E. \& Young, J.S. 2004, ApJ 533, 236

Kennicutt, R.C. 2003, PASP 115, 98

Kennicutt, R.C. 1998a, ARA\&A 36, 189

Kennicutt, R.C. 1998b, ApJ 498, 541

Moustakas, J. \& Kennicutt, R.C. 2006, ApJS 164, 81

Pérez-Gonzaĺez, et al. 2006, ApJ 648, 987

\section{Discussion}

KRUMHOLZ: In evaluating the $\Sigma_{g} / \tau_{d y n}$ form of the Schmidt law, you are on spiral arms where the epicyclic frequency is doing something interesting. How did you deal with that?

KenNiCUTT: We took a simpler approach of setting $\tau_{d y n}$ to the local orbit time, to be consistent with Kennicutt (1998b). As you suggest one could examine other formulations that would scale more closely with the local dynamical timescales, but one should bear in mind that these dynamical prescriptions for the star formation law were never intended to be applied on the local scale. There were intended as parametrizations for analytical or numerical models of galaxy formation and evolution. But given the consistency of the Schmidt law on these smaller scales it certainly makes sense to look more closely at the dynamical scaling laws.

BLITZ: You showed a very good improvement in the scatter for $\mathrm{H} \alpha$-based star formation rates with your extinction corrections applied, but this was for global emission. Have you looked at this on smaller scales, or on annular averages?

Kennicutt: Yes, to some extent. Within the SINGS project Daniela Calzetti and collaborators have analyzed about 220 individual star-forming regions in a set of SINGS galaxies for which we have infrared, $\mathrm{H} \alpha$, and $\mathrm{P} \alpha$ measurements, and those results are presented in Figure 2 here. The scatter is considerably larger than for entire galaxies, and I attribute that to object to object variations in dust geometry, cluster ages, and stochasticity in the number of $\mathrm{O}$ stars in each object, etc. That sets a physical limit to the precision of these methods for individual HII regions and clusters. 